\title{
sciendo
}

\section{Multi- to Single-Joint or the Reverse Exercise Order does not Affect Pectoralis Major Workout Performance}

\author{
by \\ Gilmar Weber Senna ${ }^{1,2,3}$, Jeffrey Michael Willardson ${ }^{4}$, Estevão Scudese ${ }^{1,2,3}$, \\ Roberto Simão ${ }^{5}$, Cristiano Queiroz de Oliveira ${ }^{3}$, Jadson de Oliveira Lima², \\ Marzo Edir Da Silva-Grigoletto ${ }^{6}$, Estélio Henrique Martin Dantas ${ }^{1,2}$
}

The purpose of this study was to examine the acute effects of multi- to single-joint or the reverse exercise order on repetition performance and perceived exertion for the pectoralis major. Fourteen trained men $(24.05 \pm 4.17$ yrs, $78.85 \pm 3.51 \mathrm{~kg}, 175.42 \pm 4.01 \mathrm{~cm}$ ) underwent two different training sequences (SEQ1 and SEQ2). In SEQ1, all subjects performed 5 sets for maximal repetitions, with a 2-min rest interval, of the bench press followed by the machine chest fly with 10 repetitions maximum load. In SEQ2, the same procedures were repeated, but with the reverse order. The $t$-test did not show any differences $(p=0.140)$ in total workout repetitions between SEQ1 $62.22 \pm 11.00$ repetitions) and SEQ2 (55.40 \pm 8.51 repetitions). Conversely, the total repetition number for the bench press exercise was significantly greater $(p=0.001)$ following SEQ1 (34.36 \pm 4.68 repetitions) compared to SEQ2 $(25.85 \pm 6.73$ repetitions). In contrast, the total repetition number for the machine chest fly exercise following SEQ2 was significantly greater $(p=0.001)(33.50+4.11$ repetitions) compared to SEQ1 (27.85 \pm 6.52 repetitions). Despite no significant differences found for the rating of perceived exertion (RPE) values between SEQ1 and SEQ2 for the barbell bench press in all sets ( $p \geq 0.083)$, significantly higher RPE values for the machine chest fly were observed over the first three sets following SEQ1 compared to SEQ2 ( $p<0.01)$. In conclusion, the total workout repetitions were not significantly different when performing the traditional multi- to single-joint or the reverse exercise order when training the pectoralis major muscle.

Key words: muscular strength, weight lifting, physical fitness, health promotion.

\section{Introduction}

The order of exercises is crucial when designing resistance exercise programs (Fleck et al., 2004). It has been established that manipulating exercise order directly affects acute repetition performance (Gentil et al., 2007; Miranda et al., 2010; Sforzo and Touey, 1996; Silva et al., 2009; Spreuwenberg et al., 2006) and strength adaptations (Dias et al., 2010), independently of other prescriptive variables. Guidelines from the American College of Sports and Medicine (ACSM, 2009) indicate that in general, large muscle groups should be trained before small muscle groups, as well as multi-joint exercises should be performed before single-joint ones within a training session. Furthermore, according to ACSM (2009) guidelines, a

\footnotetext{
1 - Nursing and Biosciences Post-Graduation Program (PPgEnfBio), Doctorate of Federal University of State of Rio de Janeiro (UNIRIO), Rio de Janeiro, Brazil.

2 - Biosciences Laboratory of Human Movement (LABIMH), Tiradentes University (UNIT), Aracaju, Brazil.

3 - Health Science Center, Catholic University of Petrópolis, Rio de Janeiro, Brazil.

4 - Health and Human Performance Department, Rocky Mountain College, Billings, Montana, USA.

5 - School of Physical Education and Sports, Federal University of Rio de Janeiro (EEFD/UFRJ), Rio de Janeiro, Brazil.

6 - Federal University of Sergipe, São Cristóvão, Brazil / Scientific Sport Association, Aracaju, Brazil.
} 
combination of both multi- and single-joint exercises should be utilized for maximizing overall muscle strength and hypertrophy.

Simão et al. (2005) indicated that exercise order within a training session should be prioritized based on the muscle group or a movement pattern needing improvement. In line with this suggestion, some acute studies (Miranda et al., 2010; Sforzo and Touey, 1996; Simão et al., 2007; 2012) observed that irrespective of the exercise type (i.e. multi- or single-joint) for the same muscle group, repetitions were higher when a given exercise was performed earlier within a resistance training session. Simão et al. (2012) demonstrated the influence of different exercise sequences and found a significant decrease in the number of repetitions when varied exercises (multi- or single-joint for different prime motors) were performed later within a training session.

Sforzo and Touey (1996) examined two different exercise sequences including the back squat, leg curl, and leg extension conducted in the aforementioned or the reverse order. Four sets were performed for each exercise with an 8 repetition maximum load and 2 to $5 \mathrm{~min}$ of rest between sets. The authors observed a significant reduction in the number of repetitions in the first set of the back squat when preceded by the leg extension and leg curl exercises.

Moreover, Spreuwenberg et al. (2006) evaluated the number of back squat repetitions before and after a whole-body resistance training session, and found a significant decline in repetition performance when the back squat was performed after the workout. Interestingly, Belezza et al. (2009) observed higher repetition performance for small to large muscle group exercise order in a sample composed of elderly subjects. Similarly, Silva et al. (2009) found that small to large muscle group exercise order promoted increases in repetition performance and perceived exertion at the end of the sequences performed by elderly women.

Observing the recent state of scientific literature regarding exercise order, there are some controversial results concerning repetition performance when utilizing large to small muscle group exercise order versus the reverse order. However, to the authors' knowledge, no study to date has examined the effects of performing different types of exercises (multi- and single- joint) for the same prime mover completed in different order on repetition performance and perceived exertion.

Therefore, the purpose of this study was to examine the acute effects of multi- to singlejoint or the reverse exercise order on repetition performance and perceived exertion for the pectoralis major. The multi- and single-joint exercises selected for this study were the barbell bench press (BP) and the machine chest fly (MCF), respectively.

\section{Methods}

\section{Participants}

Fourteen trained men with at least one year of consistent resistance training experience were selected to participate in the study $(24.05 \pm$ 4.17 years, $78.85 \pm 3.51 \mathrm{~kg}, 175.42 \pm 4.01 \mathrm{~cm}, 7.89 \pm$ $3.78 \%$ body fat content, BP relative strength: $1.31 \pm$ $0.13 \mathrm{~kg} / \mathrm{kg}^{-1}$ of body mass). The following inclusion criteria were adopted in order to standardize subjects' selection: (a) training frequency of at least four times per week, with session duration of approximately one hour; (b) non-usage of any ergogenic substance that would enhance repetition performance; (c) no acute or chronic injuries that would affect BP and MCF performance; and (d) subjects agreed not to engage in intense activity throughout test days. The sample size calculation was performed through G*Power software version 3.1 (Kiel University, 2014). The adopted variables were: a) ANOVA for repeated measurements; b) alpha error $=0.05$; c) 1 -beta error $=0.95$; number of different order conditions (2) and sets (5) verified in this study. Following the variables described above, the minimum number of participants was 12 for a power of 0.98 . Thus, the sample of 14 participants was larger than the minimum estimation required. Before data collection, all subjects answered "no" to all PAR-Q questions (Shephard, 1988). The study procedures had been previously approved by the Catholic University of Petrópolis ethics committee. In addition, all subjects read and signed an informed consent form after being informed of the testing procedures according to the Declaration of Helsinki.

\section{Measures}

After two familiarization sessions with all exercises and experimental procedures, subjects 
performed four testing sessions to determine 10RM loads for the BP and MCF. The exercise execution pattern followed previously established norms and recommendations (Baechle and Earle, 2008). On the first and third visits, BP 10-RM tests were conducted, whereas on the second and fourth visits, the MCF 10-RM tests were carried out. Each testing session was separated by 72 hours and subjects were asked not to perform any structured exercise throughout this period, as previously suggested (Senna et al., 2016; Scudese et al., 2015).

During the 10-RM tests, each subject performed a maximum of three attempts for each exercise with a 10-min rest interval in-between. The greatest load lifted over the test and retest was considered the 10-RM load. To minimize the errors in testing, the following strategies were adopted: (a) standard information concerning the testing procedures was given to the subjects before the test; (b) subjects received detailed instructions on exercise technique; (c) the body position was held constant (i.e., hand width during the $\mathrm{BP}$ ); (d) verbal encouragement was provided (McNair, 1996); and (e) the mass of all plates and bars used was determined using a precision scale.

The adult OMNI resistance exercise scale (Lagally and Robertson, 2006) was implemented to obtain the RPE values. Subjects were familiarized with the scale during their training routines one week before the initial load tests. They were asked to choose a number based on their perceived exertion or subjective intensity of effort, strain, discomfort, and/or fatigue experienced during the exercise session (Lagally and Robertson, 2006).

During the familiarization process, the $\mathrm{BP}$ and MCF were performed for 3 sets of 15 repetitions with 3-min rest intervals between sets with an estimated load based on the subject's prior resistance training experience. Immediately after each set, subjects were asked to identify their RPE values with the intent to provide a subjective measure of their perceived exertion level (Scudese et al., 2015; Senna et al., 2011, 2016).

\section{Design and Procedures}

Seventy-two hours after the last 10-RM load retest, subjects performed the first of two different training sequences (SEQ1 or SEQ2) with 72 hours between sessions. Within each sequence, subjects performed five sets until reaching concentric failure with the predetermined 10-RM loads with a 2-min rest interval between sets and exercises. A randomized within-subject design was implemented to determine the order of experimental sessions (SEQ1 or SEQ2).

In SEQ1, all subjects performed the BP followed by the MCF, while in SEQ2 the same procedures were performed, but in the reverse exercise order (the MCF followed by the BP). The warm-up before each session consisted of 2 sets of 12 repetitions at $40 \%$ of the 10-RM load (Senna et al., 2012) for the first exercise in the sequence. A 2min rest interval was implemented after the second warm-up set before the first experimental set.

All subjects were verbally encouraged to perform all five sets to concentric failure during the experimental procedure (McNair et al., 1996). No attempt was made to control the repetition velocity; however, subjects were required to use a smooth and controlled motion and a standardized range of motion. The total number of repetitions completed and the RPE (Lagally and Robertson, 2006) were recorded after each set. Both sessions were conducted at the same time of day for each subject.

\section{Statistical Analyses}

In order to verify test and retest reproducibility, the intraclass correlation coefficient (ICC) was calculated. The Student's ttest was performed to analyze differences in the total workout repetitions and total workout volume completed between sequences and for each exercise independently (the BP or the MCF). A two-way ANOVA was applied to test for an interaction effect between sequences $x$ sets performed. If necessary, further comparisons were made via Tukey post hoc tests.

To determine the magnitude of differences between sequences in repetitions performed, the effect size (ES) was evaluated for each exercise set of each sequence. Briefly, the ES of each exercise set was calculated using the number of repetitions completed in the first set (as the pre-test value) and the number of repetitions completed in the second through the fifth set (as the post-test value) along with the standard deviation of the first set (as the pre-test standard deviation). For the ES in distinct sequences, the number of repetitions completed for the initial 
exercise (as the pre-test value) and the number of repetitions completed for the same set within the other sequence (as the post-test value) along with standard deviation of the initial exercise (as the pre-test standard derivation) were utilized. The thresholds proposed by Hopkins et al. (2009) were applied in order to determine the magnitude of effects. Specifically, ES of 0.20 or less was considered trivial, from 0.21 to 0.59 small, from 0.60 to 1.19 moderate, from 1.20 to 1.99 large, from 2.0 to 3.9 very large, and $>4.0$ nearly perfect. The Friedman test was used to analyze RPE scores after each set, and the Wilcoxon test to verify differences between SEQ1 and SEQ2. If necessary, a parallel test was applied for multiple comparisons. An alpha value of $p<0.05$ was adopted to establish the significance of comparisons. The SPSS software 21.0 version was used for the statistical analyses (IBM, Inc).

\section{Results}

An excellent test/retest correlation was found for the 10-RM load using the ICC (BP, $r=$ 0.99; $\mathrm{MCF}, \mathrm{r}=0.99$ ), and no significant differences were observed between the test/retest loads via the paired t-test (BP, $p=0.164 ; \mathrm{MCF}, p=0.110)$. For total workout repetitions, the Student's t-test showed no significant differences $(p=0.140)$ between SEQ1 (62.22 \pm 11.00 repetitions) and SEQ2 (55.40 \pm 8.51 repetitions) (Figure 1). In addition, the statistical analyses for total workout volume showed no significant differences $(p=$ $0.142)$ between SEQ1 $(4933.14 \pm 1080.01$ repetitions $\mathrm{x} \mathrm{kg})$ and SEQ2 $(4719.29 \pm 794.56$ repetitions $\mathrm{x} \mathrm{kg})$.

For the BP independently, the t-test showed significant differences $(p=0.001)$ in the total number of repetitions performed between SEQ1 (34.36 \pm 4.68 repetitions) and SEQ2 (25.85 \pm 6.73 repetitions). Additionally, the data for total workout volume showed significant differences $(p=0.0001)$ between SEQ1 $(2665.28 \pm 485.89$ repetitions $\mathrm{x} \mathrm{kg}$ ) and SEQ2 (1998.57 \pm 540.61 repetitions $x \mathrm{~kg}$ ). Independently for the $\mathrm{MCF}$, the t-test analysis revealed significant differences $(p=$ 0.001 ) for the total number of repetitions performed between SEQ1 (27.85 \pm 6.52 repetitions) and SEQ2 (33.50 \pm 4.11 repetitions) (Figure 2). Furthermore, for total workout volume the t-test showed significant differences $(p=0.001)$ between SEQ1 (2267.85 \pm 633.03 repetitions $x \mathrm{~kg})$ and SEQ2 (2720.71 \pm 486.86 repetitions $x \mathrm{~kg}$ ).

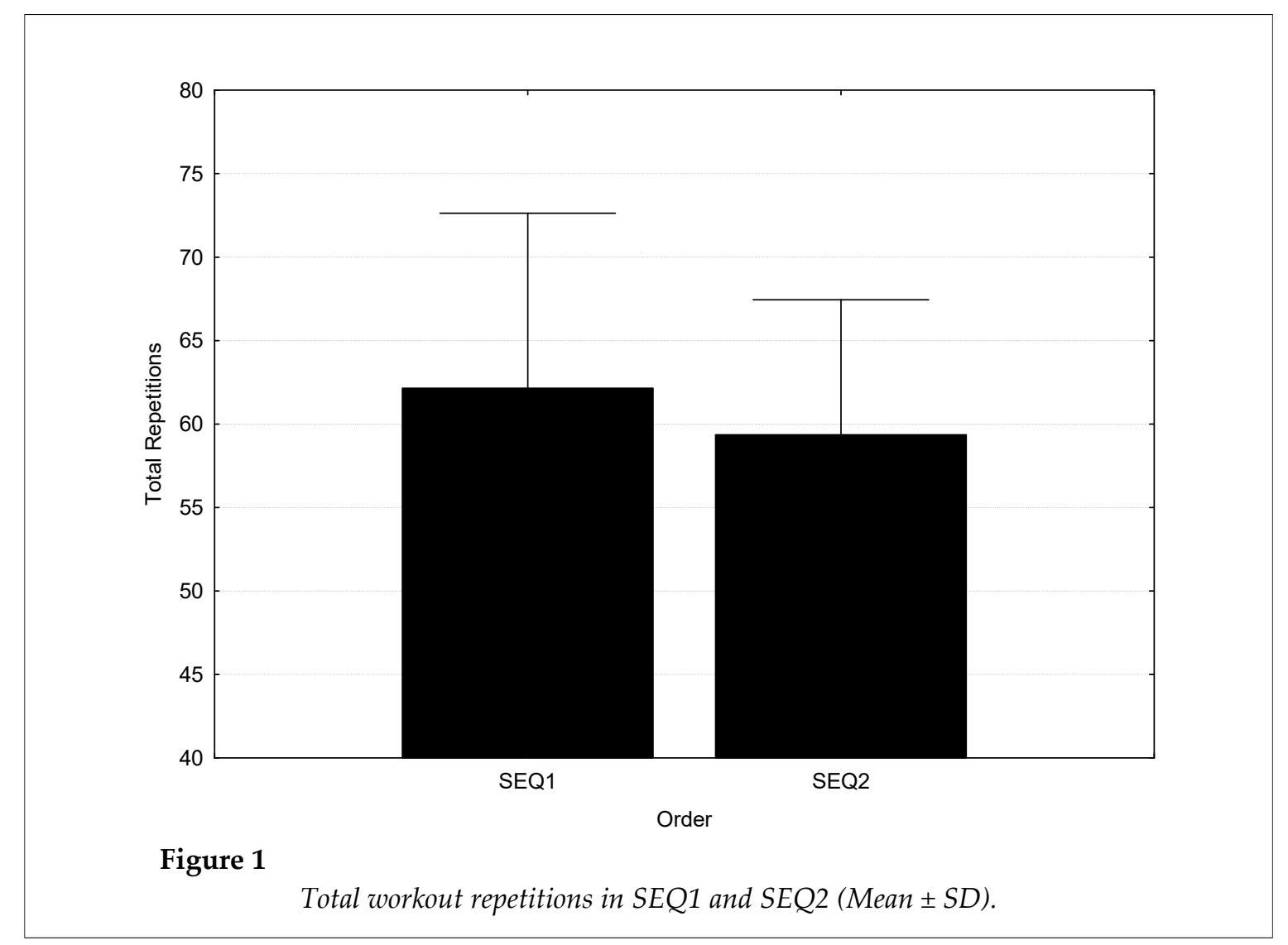




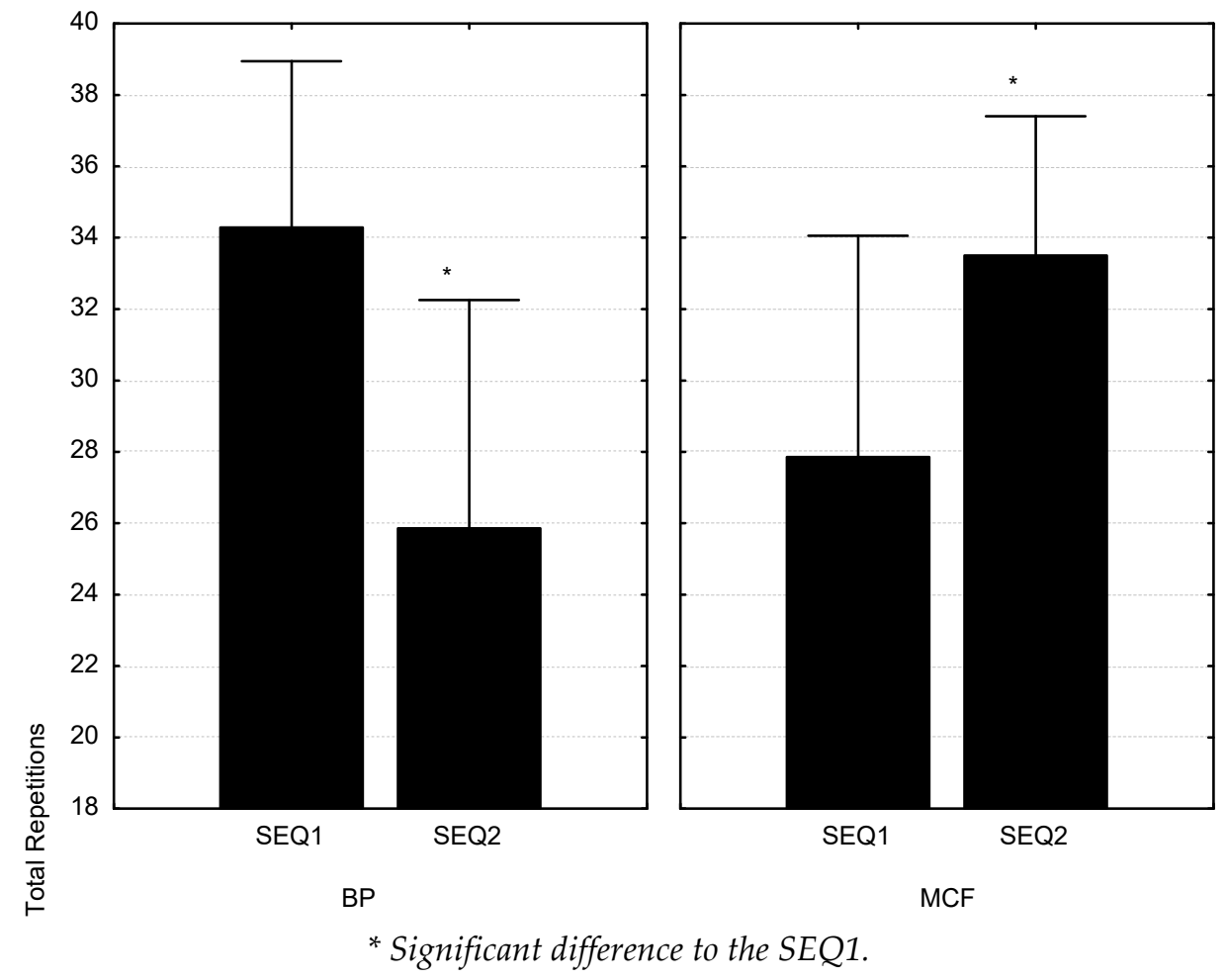

Figure 2

Total number of repetitions for each exercise independently (BP and MCF) in SEQ1 and SEQ2 (Mean \pm SD).

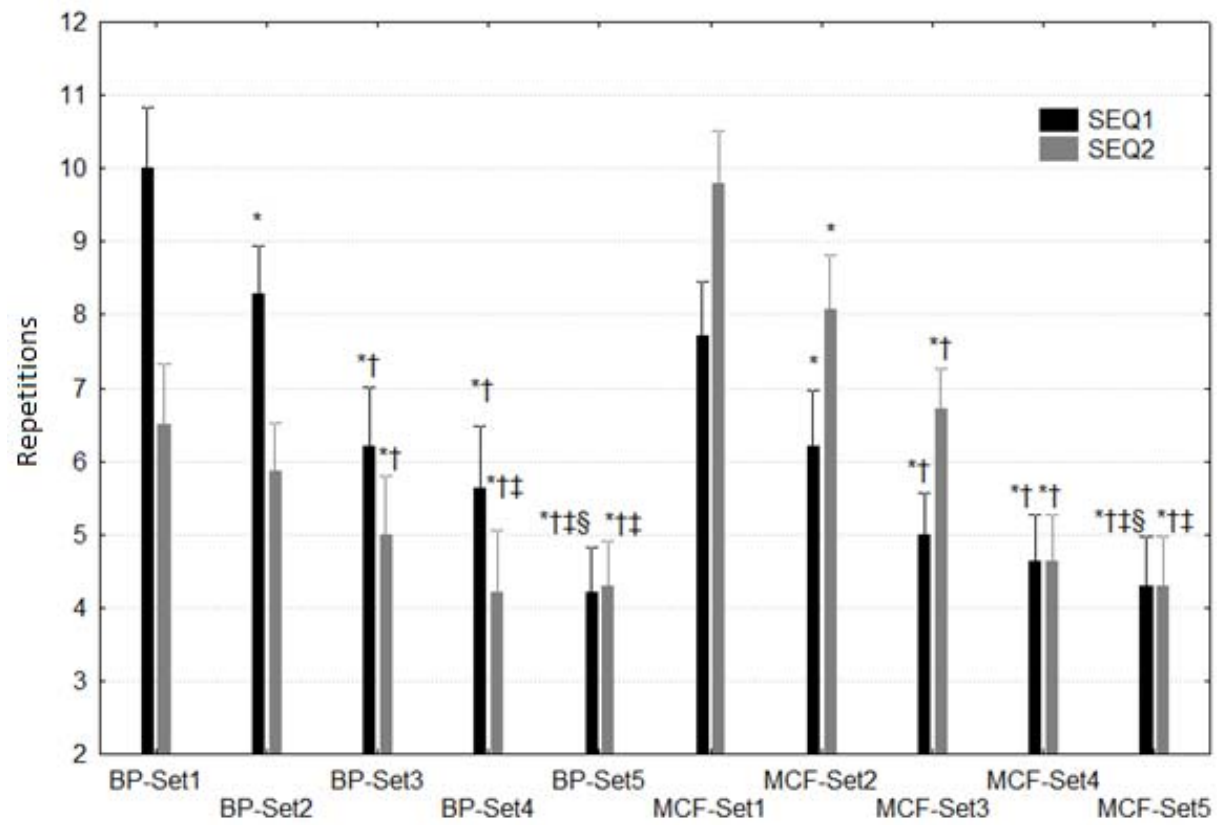

Figure 3

The number of repetition for each exercise set within each sequence

* Significant difference to the $1^{\text {st }}$ set; + Significant difference to the $2^{\text {nd }}$ set. $\neq$ Significant difference to the $3^{\text {rd }}$ set; $\S$ Significant difference to the $4^{\text {th }}$ set. 


\begin{tabular}{|c|c|c|c|c|}
\hline \multicolumn{5}{|c|}{$\begin{array}{r}\text { Table } 1 \\
\text { Effect Size values between exercise sets within SEQ1 and SEQ2. }\end{array}$} \\
\hline & Set 2 & Set 3 & Set 4 & Set 5 \\
\hline \multicolumn{5}{|c|}{ Bench Press } \\
\hline SEQ1 & $\begin{array}{c}6.15 \\
\text { (nearly perfect) }\end{array}$ & $\begin{array}{c}13.88 \\
\text { (nearly perfect) }\end{array}$ & $\begin{array}{c}16.03 \\
\text { (nearly perfect) }\end{array}$ & $\begin{array}{c}21.38 \\
\text { (nearly perfect) }\end{array}$ \\
\hline SEQ2 & 0.30 (small) & 0.70 (moderate) & 1.06 (moderate) & 1.03 (moderate) \\
\hline \multicolumn{5}{|c|}{ Machine Chest Fly } \\
\hline SEQ1 & 0.80 (moderate) & 1.46 (large) & 1.65 (large) & 1.84 (large) \\
\hline SEQ2 & $\begin{array}{c}4.02 \\
\text { (nearly perfect) }\end{array}$ & $\begin{array}{c}7.21 \\
\text { (nearly perfect) } \\
\end{array}$ & $\begin{array}{c}12.08 \\
\text { (nearly perfect) }\end{array}$ & $\begin{array}{c}12.91 \\
\text { (nearly perfect) }\end{array}$ \\
\hline
\end{tabular}

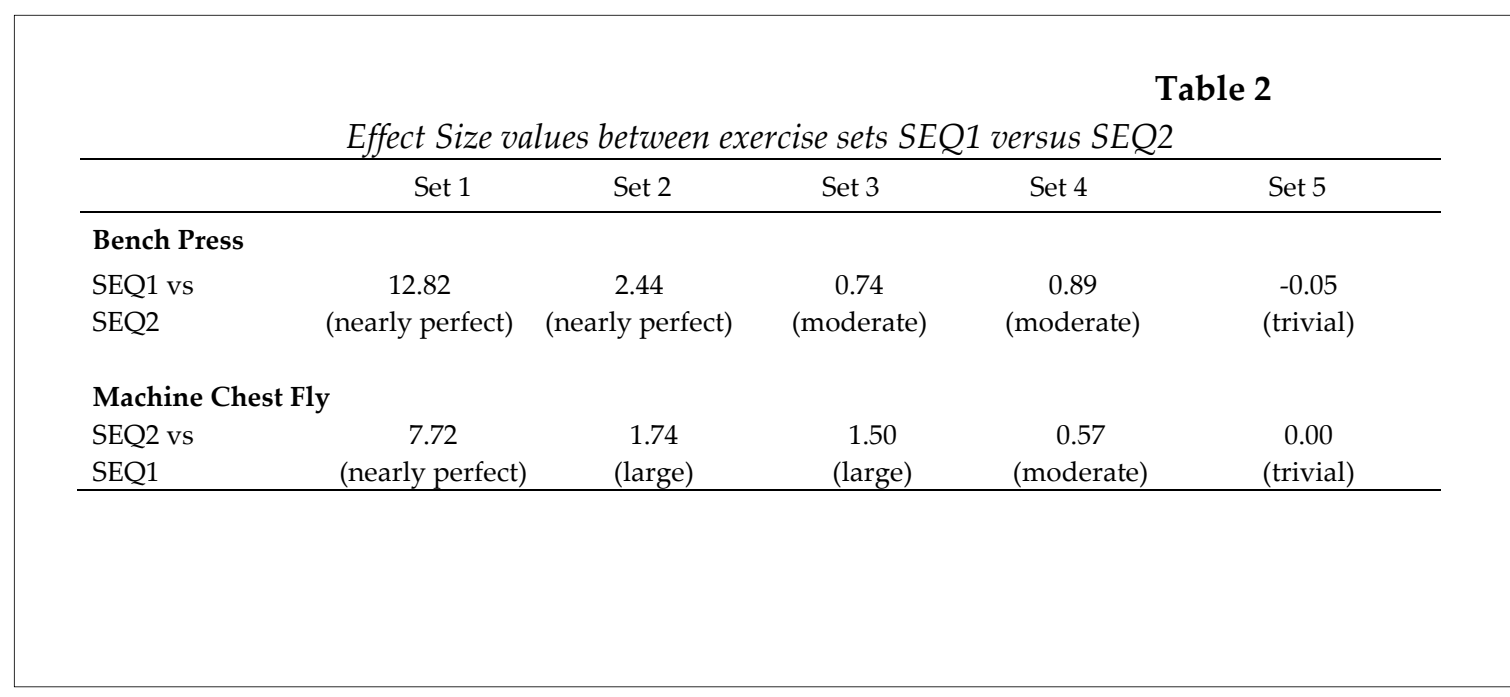

Table 3

RPE value for each exercise set in SEQ1 and SEQ2. Values are expressed in median (25-75\%).

\begin{tabular}{lccccc}
\hline & Set 1 & Set 2 & Set 3 & Set 4 & Set 5 \\
\hline Bench Press & & & & \\
SEQ1 & $6(4-7)$ & $7(6-7.5)^{*}$ & $7(7-8)^{*}$ & $8(7.25-9)^{* \#}$ & $9(8-9.5)^{* \#}$ \\
SEQ2 & $6(5-8)$ & $7(6-8.25)$ & $7.5(7-8.125)^{*}$ & $8(7-9)^{*}$ & $8.5(7-9)^{* \#}$ \\
Machine Chest Fly & & & & \\
SEQ1 & $7(7-8)$ & $8(7-8)$ & $8(8-8)$ & $8(8-9)^{*}$ & $9(9-9)^{* \#}$ \\
SEQ2 & $5(4-6)^{\mathbf{a}}$ & $6(4.25-6)^{\mathbf{a}}$ & $6(4.5-7.75)^{\mathrm{a}}$ & $7(5.25-8)^{* \#}$ & $7(5.25-8)^{* \#}$ \\
\hline
\end{tabular}

*Significantly different to Set 1; \#Significantly different to Set 2; ' Significantly different to SEQ1 
The two-way ANOVA demonstrated a significant interaction $(p=0.0001)$ for sequences $\mathrm{x}$ sets. Both sequences presented similar and progressive reductions in repetition performance for both exercises over the five sets, beginning with the second set of the first exercise (Figure 3).

The ES for the subsequent sets indicated declines of nearly perfect effect in repetition performance for both exercises when the exercise was early in the session. Specifically for the MCF, large magnitude was observed from the third set when this exercise was later in the session (Table 1). In addition, the ES between distinct sequences indicated declines of nearly perfect (first set) to small (fourth set) effects in repetition performance for each exercise (Table 2).

For the RPE values, significant differences between sets were found throughout each sequence. Specifically, for the BP, the RPE increased by the second set for SEQ1 and by the third set for SEQ2. For the MCF, the RPE increased by the fourth set for both SEQ1 and SEQ2 $(p<0.007)$. When comparing the initial three sets of the MCF exercise, the SEQ1 elicited significant increases in RPE values versus SEQ2 $(p$ $<0.01$ ). No significant differences in RPE values were observed between SEQ1 and SEQ2 for the BP $(p>0.083)$. The data for all RPE values on each sequence and exercise are presented in Table 3.

\section{Discussion}

The key finding of this study was that irrespective of the exercise sequence, the total workout repetitions were not significantly different. Specifically, for movements aimed at the pectoralis major, it did not matter for the total workout repetitions whether the barbell $\mathrm{BP}$ was followed by the MCF or the reverse order was applied. Furthermore, a progressive decrease in repetitions completed per set was observed for each exercise in both sequences. However, when independently comparing exercises between sequences, the ES evidenced a higher number of repetitions performed in the $\mathrm{BP}$ exercise for the SEQ1 (BP performed early) compared to SEQ2 (BP performed later) within the initial sets. This effect was not as evident with regard to MCF repetition performance. Therefore, the BP appears to be influenced by the effects of fatigue to a greater extent than the MCF.

The present results are somehow converse to the ACSM (2009) recommendations that suggest large before small muscle group exercises and multi- before single-joint exercises in a resistance training session. The present study demonstrated that there was no difference in total workout repetitions with either order. Simão et al. (2005) suggested that when considering acute responses or chronic adaptations, exercises and muscle parts intended to be improved should be included in the beginning of a resistance training session, irrespective of the muscle size or exercise type (multi- or single-joint).

Sforzo and Touey (1996) conducted the first experiment that focused on exercise order as an experimental variable on repetition performance. Briefly, seventeen trained men completed two sessions consisting of six exercises; four sets with an 8-RM load and 2-min rest intervals between sets. One session consisted of large (e.g. multi-joint) to small muscle group (e.g. single-joint) exercises, and the other session followed the reverse order. The results indicated that when small muscle group exercises (e.g. single-joint) preceded large muscle group exercises (e.g. multi-joint), significantly fewer repetitions were completed within large muscle group exercises. The current study did indicate that when each exercise (the $\mathrm{BP}$ and the MCF) was considered independently, there was a significant reduction in repetition performance when a given exercise was performed second in a sequence.

The present study differed from previous studies by specifically focusing on two exercises (the BP and the MCF) commonly performed for the same muscle (pectoralis major) and performed in different orders. Our results indicate that performance of the last exercise is always negatively affected independently of the exercise modality (multi- or single-joint). However, no significant difference was observed between sequences when considering the total workout repetitions. In a similar study design, Belezza et al. (2009) found that the small to large muscle group exercise sequence optimized total workout repetition performance in a group of elderly participants. It might be that in the elderly participants, performing smaller muscle group exercises first served as a warm-up to facilitate a greater number of repetitions within larger muscle group exercises.

RPE values have been used for assessing 
the relative intensity of resistance exercises (Lagally and Robertson, 2006). Prior studies (Belezza et al., 2009; Simão et al., 2005, 2007) reported no significant difference in RPE values between distinct sequences (large to small or small to large muscle group exercises), suggesting that the exercise order had no influence on the RPE. In the present study, RPE values significantly increased over subsequent sets. In addition, there were no significant differences in RPE values for the BP between sequences. However, for the MCF, there was a significant decrease observed in SEQ2 versus SEQ1. These data suggest that for a single-joint exercise like the $\mathrm{MCF}$, performing it later in a training sequence, promoted greater subjective effort when compared to the reverse order, whereas for the BP, there was no effect of exercise order on subjective effort sensation.

One of the possible concerns regarding this experiment would be the lack of control on repetition velocity during both exercise performance. However, this type of control might have triggered other experimental problems when testing maximum strength. For instance, repetition velocity control would affect the performance sooner than expected once the sets were performed to the maximum according to procedures of strength testing for repetition maximum recommended by the ACSM guidelines (ACSM, 2014). For example, if any given subject had mismatched the repetitions with the preset timing due to fatigue, the test would have to be interrupted. In addition, in none of the previous studies focused on exercise order and acute performance such a type of control has been exerted.

This study adds to the growing body of knowledge with regard to the importance of programming the exercise order. The results indicate that multi- and single-joint exercise order does not affect the total number of repetitions completed in a resistance training session when working the same muscle group. Strength coaches can apply the results of this study in order to improve the efficiency and effectiveness of a resistance exercise program. From a practical perspective, those exercises that are most important (based on individual needs) should be placed early in a resistance training session regardless of whether they are multi- or singlejoint exercises. However, it seems that total workout repetitions are not significantly different when performing the traditional multi- to singlejoint or the reverse order when training the pectoralis major.

\section{References}

American College of Sports Medicine. Position stand on progression models in resistance exercise for healthy adults. Med Sci Sports Exerc, 2009; 41: 687-708

Baechle TR, Earle RW. Essentials of strength training and conditioning. (3nd ed.). Illinois: Human Kinetics; 2008

Bellezza PA, Hall EE, Miller PC, Bixby WR. The influence of exercise order on blood lactate, perceptual, and affective responses. J Strength Cond Res, 2009; 23: 203-208

Dias I, de Salles BF, Novaes J, Costa PB, Simão R. Influence of exercise order on maximum strength in untrained young men. J Sci Med Sport, 2010; 13: 65-69

Fleck SJ, Kraemer WJ. Designing Resistance Training Programs. Champaign: Human Kinetics; 2004

Gentil P, Oliveira E, Rocha Júnior VA, Carmo J, Bottaro M. Effects of exercise order on upper-body muscle activation and exercise performance. J Strength Cond Res, 2007; 21: 1082-1086

Hopkins WG, Marshall SW, Batterham AM, Hanin J. Progressive statistics for studies in sports medicine and exercise science. Med Sci Sports Exerc, 2009; 41: 3-12

Lagally KM, Robertson RJ. Construct validity of the OMNI resistance exercise scale. J Strength Cond Res, 2006; 20: 252-256

McNair PJ, Depledge J, Brettkelly M, Stanley SN. Verbal encouragement: effect on maximum effort voluntary muscle action. Br J Sports Med, 1996; 30: 243-245 
Miranda H, Simão R, Vigário PS, de Salles BF, Pacheco MT, Willardson JM. Exercise order interacts with rest interval during upper-body resistance exercise. J Strength Cond Res, 2010; 24: 1573-1577

Scudese E, Willardson JM, Simão R, de Salles BF, Senna G, Miranda H. The effect of rest interval length on repetition consistency and perceived exertion during near maximal loaded bench press sets. J Strength Cond Res, 2015; 29: 3079-3083

Senna G, Figueiredo T, Scudese E, Baffi M, Carneiro F, Moraes E, Miranda H, Simão R. Influence of different rest interval length in multi-joint and single-joint exercises on repetition performance, perceived exertion, and blood lactate. J Exerc Physiol Online, 2012; 15: 96-106

Senna G, Willardson JM, de Salles BF, Scudese E, Palma A, Simão R. The effect of rest interval length on multi and single-joint exercise performance and perceived exertion. J Strength Cond Res, 2011; 25: 31573162

Senna G, Willardson JM, Scudese E, Simão R, Queiroz C, Avelar R, Dantas EHM. Effect of different inter-set rest intervals on performance of single and multijoint exercises with near-maximal loads. J Strength Cond Res, 2016; 30: 710-716

Sforzo GA, Touey PR. Manipulating exercise order affects muscular performance during a resistance exercise training session. J Strength Cond Res, 1996; 10: 20-24

Shephard RJ. PAR-Q, Canadian home fitness test and exercise screening alternatives. Sports Med, 1988; 5: 185-195

Silva NSL, Monteiro WD, Farinatti PTV. Influence of exercise order on the number of repetitions and perceived exertion in young and older women. Braz J Sports Med, 2009; 15: 219-23

Simão R, Farinatti PTV, Polito MD, Maior AS, Fleck SJ. Influence of exercise order on the number of repetitions performed and perceived during resistive exercises. J Strength Cond Res, 2005; 19: 152-156

Simão R, Farinatti PTV, Polito MD, Viveiros L, Fleck SJ. Influence of exercise order on the number of repetitions performed and perceived exertion during resistance exercise in women. J Strength Cond Res, 2007; 21: 23-28

Simão R, de Salles BF, Figueiredo T, Dias I, Willardson JM. Exercise order in resistance training. Sports Med, 2012; 42: 251-265

Spreuwenberg LPB, Kraemer WJ, Spiering BA, Volek JS, Hatfield DL, Silvestre R, Vingren JL, Fragala MS, Hakkinen K, Newton RU, Maresh CM, Fleck SJ. Influence of exercise order in a resistance-training exercise session. J Strength Cond Res, 2006; 20: 141-144

\section{Corresponding author:}

\section{Gilmar Weber Senna, Ph.D.}

Federal University of State of Rio de Janeiro (UNIRIO)

Xavier Sigaud street - 290 - 301 - Praia Vermelha

ZipCode: 22290-180 - Rio de Janeiro - RJ - Brazil.

E-mail: sennagw@gmail.com 